\title{
ACTIONS OF ELEMENTARY P-GROUPS ON MANIFOLDS
}

\author{
BY \\ L. N. MANN AND J. C. SU
}

1. Introduction. An elementary p-group of rank $k$ will be a group isomorphic to $k$ copies of $Z_{p}$ (the additive group of integers modulo a prime $p$ ). Smith [7] has studied the effective actions of such groups on the $n$-sphere $S^{n}$ and has shown that $k \leqq(n+1) / 2$ for $p \neq 2$ and $k \leqq n+1$ for $p=2$. Free actions of elementary $p$-groups on products of spheres have been investigated by Conner [4] and Heller [5] and again appropriate bounds for $k$ have been found. In the first part of this paper we establish a result which states that given a compact manifold $X$ of dimension $n$, the sum of whose $\bmod p$ Betti numbers is an integer $B$, there exists an integer $N(n, B)$, depending only upon $n$ and $B$, such that no elementary $p$-group of rank greater than $N$ can operate effectively on $X$.

In the second part of this paper we turn our attention to effective actions of elementary 2-groups on projective spaces. We establish "best possible" bounds for the ranks of such groups. It is of particular interest to note that the projective line and projective 3-space are singled out from the other projective spaces in our Theorem 3.5. Finally we turn our attention to actions of elementary p-groups on lens spaces. As might be expected, we deal with cohomology manifolds rather than with manifolds in this paper. The authors are grateful to Professors E. E. Floyd and E. C. Paige for their encouragement and helpful suggestions.

2. General results. An action of a transformation group $G$ on a space $X$ is said to be effective if the identity of $G$ is the only element of $G$ which leaves $X$ pointwise fixed. An isotropy subgroup $G_{x_{0}}$ at a point $x_{0}$ in $X$ is defined to be the set of all elements of $G$ which leave $x_{0}$ fixed. Finally the action of $G$ on $X$ is said to be free if the only isotropy subgroup of $G$ is the identity element.

Unless stated otherwise, all spaces considered will be locally compact Hausdorff. For such a space $X$ we shall use the Alexander-Wallace-Spanier (AWS) cohomology with compact supports and coefficient group $Z_{p}$ ( $p$, prime). Most of our main results, however, will assume the compactness of the space. For a compact space, the AWS and usual Čech cohomology coincide. In any case we shall denote the $i$ th cohomology group simply by $H^{i}(X)$. Our definition of a cohomology $n$-manifold mod $p$ will be that given in [1]. Intuitively one may consider a cohomology $n$-manifold mod $p$ to be a locally compact Hausdorff

Received by the editors December 18, 1961. 
space which has a local cohomology structure (coefficient group $Z_{p}$ ) resembling that of euclidean $n$-space. We have the following theorem of Borel's [1, XIII, 4.3]

Proposition 2.1 (BoreL). Let $G$ be an elementary p-group operating on a first countable cohomology $n$-manifold $X \bmod p$. Let $x \in X$ be a fixed point of $G$ on $X$ and let $n(H)$ be the cohomology dimension mod $p$ of the component of $x$ in the fixed point set of a subgroup $H$ of $G$. If $r=n(G)$, we have

$$
n-r=\sum_{H}(n(H)-r)
$$

where $H$ runs through the subgroups of $G$ of index $p$.

Borel's theorem enables us to establish easily the following result. For a space $Y, \operatorname{dim}_{p} Y$ will denote the cohomology dimension of $Y$ modulo the coefficient group $Z_{p}[1, I, 1.2]$.

THEOREM 2.2. Let $G$ be an elementary p-group of rank $k$ operating effectively on a first countable connected cohomology n-manifold $X \bmod p$. Suppose $\operatorname{dim}_{p} F(G)=r \geqq 0$ where $F(G)$ is the fixed point set of $G$ on $X$. Then

$$
k \leqq \begin{cases}\frac{n-r}{2} & \text { for } p \neq 2 \\ n-r & \text { for } p=2\end{cases}
$$

Proof. We shall prove the theorem for $p$ odd; for $p=2$ a similar proof will hold. Since $r \geqq 0$, there exist a point $x$ in $F(G)$. Using the notation of (2.1) and assuming $n(G)=r$, we have $n-r>0[1, \mathrm{I}, 4.6]$. By (2.1), there must exist a subgroup $H_{1} \subset G$ of rank $k-1$ such that $r_{1}=n\left(H_{1}\right)>n(G)=r$. Due to parity restrictions $[1, \mathrm{~V}, 2.3], n-r_{1}$ and $n-r$ are even; hence $r_{1}-r$ is even and $r_{1} \geqq r+2$. Now consider the effective action of $H_{1}$ on $X$. By (2.1) again, there exists a subgroup $H_{2}$ of $H_{1}$ of rank $k-2$ such that $r_{2}=n\left(H_{2}\right)>n\left(H_{1}\right)=r_{1}$. By parity again, $r_{2} \geqq r_{1}+2$. Continuing in this manner we finally obtain a subgroup $H_{k-1}$ of rank 1 such that $r_{k-1}=n\left(H_{k-1}\right) \geqq n\left(H_{k-2}\right)+2=r_{k-2}+2$. We have

$$
n \geqq r_{k-1}+2 \geqq\{r+2(k-1)\}+2=r+2 k
$$

Hence

$$
k \leqq \frac{n-r}{2}
$$

Turning to free actions of elementary p-groups, we establish a result which is essentially due to Heller [5]. However, as we shall be interested in applications in the setting of AWS cohomology rather than singular homology, we present the following reformulation of Heller's result. Our proof is based on a spectral sequence of R. G. Swan [11]. 
THEOREM 2.3. Let $G$ be an elementary p-group of rank $k$ acting freely on a compact connected space $X$. Assume that $H^{*}(X)$ is finite dimensional; i.e., there exists $n$ such that $H^{i}(X)=0$ for all $i>n$ and $\operatorname{dim} H^{i}(X)=b_{i}$ is finite for all $0 \leqq i \leqq n$. Let $B=\sum_{i=0}^{n} b_{i}$. Then there exists an integer $f(n, B)$, depending on $n$ and $B$ only, such that $k \leqq f(n, B)$.

Proof. According to [11, Theorem 3.1], there exists a convergent spectral sequence $\left(E_{r}\right)$ whose $E_{2}$-term is given by $E_{2}^{s, t}=\hat{H}^{s}\left(G ; H^{t}(X)\right)$ and whose $E_{\infty}$-term is trivial, where $\hat{H}^{s}\left(G ; H^{t}(X)\right)$ is the Tate cohomology group [3] of $G$. Let $s \geqq 0$ be any integer. We have $E_{r+1}^{s+1,0}=E_{r}^{s+1,0} / \operatorname{Im}\left(E_{r}^{s+1-r, r-1} \rightarrow^{d r} E_{r}^{s+1,0}\right)$ for all $r \geqq 2$. Hence

or

$$
\begin{aligned}
\operatorname{dim} E_{r+1}^{s+1,0} & \geqq \operatorname{dim} E_{r}^{s+1,0}-\operatorname{dim} E_{r}^{s+1-r, r-1} \\
& \geqq \operatorname{dim} E_{r}^{s+1,0}-\operatorname{dim} E_{2}^{s+1-r, r-1}
\end{aligned}
$$

$$
\operatorname{dim} E_{2}^{s+1-r, r-1} \geqq \operatorname{dim} E_{r}^{s+1,0}-\operatorname{dim} E_{r+1}^{s+1,0} \quad \text { for all } r \geqq 2 .
$$

The condition $H^{i}(X)=0$ for all $i>n$ implies that $E_{r}^{s+1,0}=E_{\infty}^{s+1,0}=0$ for all $r>n+1$. Hence from (1) we obtain

$$
\operatorname{dim} E_{2}^{s+1,0} \leqq \sum_{j=1}^{n} \operatorname{dim} E_{2}^{s-j, j} \quad \text { for all } s \geqq 0 .
$$

By Kunneth's formula, it is easy to verify that

$$
\operatorname{dim} E_{2}^{s, t} \leqq \begin{cases}\left(\begin{array}{c}
s+k-1 \\
k-1
\end{array}\right) \operatorname{dim} H^{t}(X) & \text { if } s \geqq 0, \\
\left(\begin{array}{c}
-s-1+k-1 \\
k-1
\end{array}\right) \operatorname{dim} H^{t}(X) & \text { if } s<0\end{cases}
$$

where

$$
\left(\begin{array}{c}
m \\
n
\end{array}\right)=\frac{m !}{n !(m-n) !}
$$

is the binomial coefficient. Moreover, equality holds if $G$ acts trivially on $H^{t}(X)$. Using this, (2) becomes

$$
\left(\begin{array}{l}
s+k \\
k-1
\end{array}\right) \leqq \sum_{j=1}^{s}\left(\begin{array}{c}
s-j+k-1 \\
k-1
\end{array}\right) b_{j}+\sum_{j=s+1}^{n}\left(\begin{array}{c}
j-s+k-2 \\
k-1
\end{array}\right) b_{j} \text { for all } s \geqq 0 .
$$

Taking $s=n$, we obtain

$$
\left(\begin{array}{c}
n+k \\
k-1
\end{array}\right) \leqq\left\{\sum_{j=0}^{n-1}\left(\begin{array}{c}
j+k-1 \\
k-1
\end{array}\right)\right\} \max _{j} b_{j}=\left(\begin{array}{c}
n+k-1 \\
k
\end{array}\right) \max _{j} b_{j} .
$$

Simplifying, 


$$
k^{2}+n k \leqq n(n+1) \max _{j} b_{j} \leqq n(n+1) B .
$$

The assertion of the theorem now follows by taking, for example

$$
f(n, B)=\frac{\left(n^{2}+4 n(n+1) B\right)^{1 / 2}-n}{2} .
$$

The inequality (3) may be found in [5], where the $b_{j}$ 's represent Betti numbers in the singular sense. The inequality in [5] seems to have a slight error.

Having established the above result, it is reasonable to ask if there is a bound depending only upon the dimension of a space for the number of copies of $Z_{p}$ which can operate freely upon it (i.e., was it really necessary to include $B$ in the bound $f(n, B)$ ?). We may settle this question by showing that for each integer $k$ there exists a compact orientable 2-manifold which admits a free action of an elementary $p$-group ( $p$, any prime) of rank $k(1)$. The following is due to J. M. Kister. Let $Y$ be a 2-sphere with $k$ handles. Then $H_{1}(Y)$ is isomorphic to $2 k$ copies of the integers, and for each prime $p$ there exists a subgroup $H$ of $H_{1}(Y)$ such that the quotient group $H_{1}(Y) / H$ is an elementary $p$-group of rank $2 k$. There is a homomorphism of the fundamental group $\pi_{1}(Y)$ onto $H_{1}(Y) / H$. Letting $N$ be the kernel of this homomorphism, $N$ is normal in $\pi_{1}(Y)$ and $\pi_{1}(Y) / N$ $\cong H_{1}(Y) / H$. Let $X$ be the universal covering space of $Y$. Then $\pi_{1}(Y)$ operates freely on $X$ and, in particular, the subgroup $N$ operators freely on $X$. Hence $\pi_{1}(Y) / N$ operates freely on the orbit space $X / N$. Finally we observe that $X / N$ is an orientable 2-manifold (it is a finite covering space of $Y$ ) and $\pi_{1}(Y) / N$ is an elementary $p$-group of rank $2 k$. It may be verified that $X / N$ is a sphere with $p^{2 k}(k-1)+1$ handles.

With the following combinatorial lemma we shall be able to handle the general case of an effective action of an elementary $p$-group on a compact manifold.

LEMMA 2.4. Let a finite transformation group $G$ of order s operate (not necessarily effectively) on a space $X$. Suppose that for the integer $m, X$ contains $M$ components of dimension $m$ and let $C$ be one such component. Then there exists a subgroup $H$ of $G$ of order at least $s / M$ which leaves $C$ invariant.

Proof. Order the $m$-dimensional components of $X, C=C_{1}, C_{2}, \ldots, C_{M}$. Since each $g$ in $G$ is a homeomorphism on $X$, the $C_{j}$ are permuted among themselves by $G$. Consider the set $\{g C, g \in G\}$. Since there are $s g$-images of $C$ and $M$ components, there must exist at least $s / M$ distinct elements of $g$, say $g_{1}, \ldots, g_{t}$ $(t \geqq s / M)$, such that $g_{i} C=C_{j}$ for some $j ; i=1,2, \ldots, t$. Now let $H$ be the subgroup of $G$ which leaves $C$ invariant. Consider the elements $g_{1}^{-1} g_{1}, g_{1}^{-1} g_{2}, \ldots$, $g_{1}^{-1} g_{t}$. These $t$ elements of $G$ are all distinct and are, in fact, elements of $H$. Since $t \geqq s / M$, the proof is complete.

(1) Actually R. D. Anderson (Pacific J. Math. 7 (1957), 797-810). has shown that every finite group operates freely on some compact orientable 2-manifold. 
THEOREM 2.5. Let $G$ be an elementary p-group of rank $k$ operating effectively on a compact first countable connected cohomology $n$-manifold $X \bmod p$. Let $B_{X}$ be the finite integer representing the sum of the $\bmod p$ Betti numbers of $X$. Then

(1) If $\operatorname{dim}_{p} F(G)=r \geqq 0$,

$$
k \leqq \begin{cases}\frac{n-r}{2} & \text { for } p \neq 2 \\ n-r & \text { for } p=2 .\end{cases}
$$

(2) If $G$ operates freely on $X$,

$$
k \leqq f\left(n, B_{X}\right)
$$

where $f\left(n, B_{X}\right)$ is the bound of $(2.3)$.

(3) Otherwise,

$$
k \leqq \begin{cases}f\left(m, B_{X}\right)+\frac{n-m}{2}+\log _{p} B_{X} & \text { for } p \neq 2, \\ f\left(m, B_{X}\right)+(n-m)+\log _{2} B_{X} & \text { for } p=2,\end{cases}
$$

where $m=\operatorname{dim}_{p} F(H)$ for $H$ a maximal isotropy subgroup of $G$.

Proof. Cases (1) and (2) are of course covered by (2.2) and (2.3). We turn to (3) and assume $p \neq 2$. Let $H$ be a maximal isotropy subgroup (i.e., $H$ is not a proper subgroup of any isotropy subgroup) of rank, say $l$. By (1) we have $l \leqq(n-m) / 2$ where $m=\operatorname{dim}_{p} F(H)$. Since $H$ is maximal and $F(G)$ is empty, $G^{\prime}=G / H$ operates freely on $F(H)$.

By an easy induction on a result of Floyd [1, III, 4.3], $B_{F(H)} \leqq B_{X}$ and $F(H)$ consists of at most $B_{F(H)}$ components. Suppose there are $M$ components of $F(H)$ which are of maximal mod $p$ dimension $m$. Let $C$ be one of these components such that $B_{C} \leqq B_{F(H)} / M$. Now $G^{\prime}$ leaves $F(H)$ invariant and $G^{\prime}$ is of order $p^{k-l}$. By (2.4), there exists a subgroup $H^{\prime}$ of $G^{\prime}$ of order at least $p^{k-l} / M$ which leaves $C$

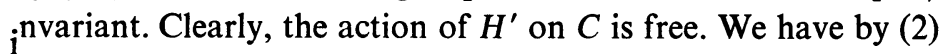

$$
\log _{p}\left(p^{k-l} / M\right) \leqq \operatorname{rank} H^{\prime} \leqq f\left(m, B_{c}\right) .
$$

Hence

$$
(k-l)-\log _{p} M \leqq f\left(m, B_{c}\right)
$$

and finally,

$$
k \leqq f\left(m, B_{c}\right)+\frac{n-m}{2}+\log _{p} M .
$$

Since $B_{C} \leqq B_{F(H)} / M$ and $M \leqq B_{F(H)} \leqq B_{X}$, we have a slightly stronger result than (3).

It is interesting to note that Theorem 2.5 is false if one drops the requirement that $X$ be a cohomology manifold. In fact, there exists for each integer $k$ a finite contractible 2-complex with an effective action of an elementary $p$-group of rank $k$ on the complex. For this purpose, simply consider $k$ disjoint closed 2-discs joined 
together by a line passing through their centers. The group action is defined by having the $i$ th copy of $Z_{p}$ in the group act as a group of rotations on the $i$ th disc while leaving all other points of the complex stationary.

3. Projective spaces. A cohomology projective $n$-space will be a compact space which has the mod 2 cohomology ring structure of real projective $n$-space, $P(n)$. A generalized cohomology projective $n$-space will be a cohomology projective $n$-space which is also a cohomology manifold mod 2 . It is easy to verify that a generalized cohomology projective $n$-space is in fact $n$-dimensional as a cohomology manifold mod 2. By a result of Su [8] it follows that the fixed point set of every action of $Z_{2}$ on a (generalized) cohomology projective $n$-space is either empty or consists of precisely two components, each a (generalized) cohomology projective $n_{j}$-space $(j=1,2)$ with $n_{1}+n_{2}=n-1$. We first investigate free actions of elementary 2-groups on cohomology projective spaces.

It is well known that if a finite group $G$ acts freely on an integral cohomology $n$-sphere, then the cohomology group $H^{i}(G ; Z)$ has a period $n+1[3 ; 7]$. This condition places a severe restriction upon the structure of $G$. For instance, it implies that if $G$ is a $p$-group, (a group of order a power of $p$, not necessarily abelian) then $G$ is either cyclic or is a generalized quaternion group [3]. (A generalized quaternion group is a 2-group of order $2^{r}$ with two generators, $s$ and $t$, satisfying the relations $s^{2^{r-2}}=t^{2}$ and $s t s=t$.) For our purpose, we need to consider the free actions of a $p$-group $G$ on a mod $p$ cohomology $n$-sphere.

Proposition 3.1. If a p-group $G$ acts freely on a mod $p$ cohomology $n$-sphere $X$, then $G$ is either a cyclic group or a generalized quaternion group.

Proof. The center of $G$ is nontrivial. Hence $G$ contains a subgroup isomorphic to $Z_{p}$. We show this is the only subgroup of order $p$ in $G$. Suppose $G$ contains a subgroup $H$ isomorphic to $Z_{p}^{2}=Z_{p} \oplus Z_{p}$. Now $H$ acts freely on $X$. Hence there exists a spectral sequence $\left(E_{r}\right)$ such that $E_{2}^{s, t}=H^{s}\left(Z_{p}^{2} ; H^{t}\left(X ; Z_{p}\right)\right)$ and $E_{\infty}$ is associated with $H^{*}\left(X / Z_{p}^{2} ; Z_{p}\right)$, where of course in $E_{2}^{s, t}, Z_{p}^{2}$ acts trivially on, $H^{t}\left(X ; Z_{p}\right)$. Since $E_{2}^{s, t}=0$ for $t \neq 0, n$, we have Gysin's exact sequence

$$
\ldots \rightarrow E_{2}^{s, 0} \rightarrow H^{s}\left(X / Z_{p}^{2} ; Z_{p}\right) \rightarrow E_{2}^{s-n, n} \rightarrow E_{2}^{s+1,0} \rightarrow \ldots .
$$

Since $H^{s}\left(X / Z_{p}^{2} ; Z_{p}\right)=0$ for all $s>n[6]$, we conclude immediately that $H^{i}\left(Z_{p}^{2} ; Z_{p}\right)$ is isomorphic to $H^{i+n+1}\left(Z_{p}^{2} ; Z_{p}\right)$ for all $i>0$. On the other hand, for the trivial action of $Z_{p}^{2}$ on $Z_{p}$, the group $H^{i}\left(Z_{p}^{2} ; Z_{p}\right)$ can be calculated explicitly, obtaining $\operatorname{dim} H^{i}\left(Z_{p}^{2} ; Z_{p}\right)=i+1$ which is a contradiction. Thus $G$ is a $p$-group with only one subgroup of order $p$. But it is then known [12] that such a group must be either cyclic or a generalized quaternion group.

Recall that an extension of $G$ by an abelian kernel $A$ is an exact sequence $0 \rightarrow A \rightarrow \widetilde{G} \rightarrow G \rightarrow 1$ (for this notation, see [2]) such that $A$ is an abelian normal subgroup of $G$. The set of all extensions (up to equivalence) of $G$ by $A$ is in one to one correspondence with the elements of the second cohomologyrgroup 
$H^{2}(G ; A)$, [2]. If $\tilde{G}$ acts on some space $\tilde{X}$, then so does $A$ and there is a natural induced action of $\tilde{G} / A=G$ on $\tilde{X} / A$.

Consider a principal bundle $(\tilde{X}, X, A, \pi)$, where the structure group $A$ is isomorphic to $Z_{p}$, and a nonzero element $\alpha \in H^{1}\left(X ; Z_{p}\right)$. Following [9], we call the system $(\tilde{X}, X, A, \pi)$ a cohomology covering space with respect to $\alpha$ if both $\tilde{X}$ and $X$ are connected and $\pi^{*}: H^{1}\left(X ; Z_{p}\right) \rightarrow H^{1}\left(\tilde{X} ; Z_{p}\right)$ takes $\alpha$ into zero. If $g: X \rightarrow X$ is a homeomorphism such that $g^{*}(\alpha)=\alpha$ and $\tilde{x}$ and $\tilde{x}^{\prime}$ are any two pre-assigned points in $\pi^{-1}(x)$ and $\pi^{-1}(g(x))$ respectively, then there exists a unique lifting homeomorphism $\tilde{g}: \tilde{X} \rightarrow \tilde{X}$ such that $\tilde{g}(\tilde{x})=\tilde{x}^{\prime}$ and $\pi \circ \tilde{g}=g \circ \pi$. In particular, a homeomorphism $\tilde{g}: \tilde{X} \rightarrow \tilde{X}$ is in $A$ (i.e., $\tilde{g}$ coincides with the action of some element of $A$ on $\tilde{X}$ ) if and only if $\pi \circ \tilde{g}=\pi$.

THEOREM 3.2. Let $(\tilde{X}, X, A, \pi), A$ isomorphic to $Z_{p}$, be a cohomology covering space with respect to a nonzero element $\alpha \in H^{1}\left(X ; Z_{p}\right)$ and let $G$ be a finite group acting on $X$. Suppose moreover that $g^{*}(\alpha)=\alpha$ for all $g \in G$. Then there exists an extension $0 \rightarrow A \rightarrow \tilde{G} \rightarrow G \rightarrow 1$ of $G$ by $A$ and an action of $\tilde{G}$ on $\tilde{X}$ such that the induced action of $\tilde{G} / A=G$ on $\tilde{X} / A=X$ coincides with the action of $G$ on $X$. Furthermore, if $G$ acts freely on $X$, then $\tilde{G}$ acts freely on $\tilde{X}$.

Proof. Choose a point $b \in X$ and $\tilde{b} \in \pi^{-1}(b)$. For each $g \in G$, choose a point $\tilde{x}_{g} \in \pi^{-1}(g(b))$ and agree that $\tilde{x}_{e}=\tilde{b}$, where $e$ is the identity of $G$. Then for each $g \in G$, there is a unique homeomorphism $\tilde{g}: \tilde{X} \rightarrow \tilde{X}$ such that $\pi \circ \tilde{g}=g \circ \pi$ and $\tilde{g}(\tilde{b})=\tilde{x}_{g}$. If $\sigma \in A$, then $\pi \circ \tilde{g} \circ \sigma \circ \tilde{g}^{-1}=\pi$; hence $\tilde{g} \circ \sigma \circ \tilde{g}^{-1} \in A$. In fact, $g \sigma$ $=\tilde{g} \circ \sigma \circ \tilde{g}^{-1}$ defines $A$ as a $G$-module (i.e., $G$ acts on $A$ as a group of automorphisms). For $g_{1}$ and $g_{2}$ in $G$, we have $\pi \circ \tilde{g}_{1} \circ \tilde{g}_{2} \circ\left(\left(g_{1} g_{2}\right)^{\sim}\right)^{-1}=g_{1} g_{2}\left(g_{1} g_{2}\right)^{-1}$ $\circ \pi=\pi$. Hence there is a unique element $f\left(g_{1}, g_{2}\right) \in A$ such that

$$
\tilde{g_{1}} \circ \tilde{g_{2}}=f\left(g_{1}, g_{2}\right) \circ\left(g_{1} g_{2}\right)^{\sim} \text {. }
$$

The map $f: G \times G \rightarrow A$ given by $\left(g_{1}, g_{2}\right) \rightarrow f\left(g_{1}, g_{2}\right)$ defines a 2-cochain of $G$ into $A$. $f$ is in fact a 2-cocycle. From (1) we have

$$
\tilde{g}_{1} \circ \tilde{g}_{2} \circ \tilde{g}_{3}=f\left(g_{1}, g_{2}\right) \circ\left(g_{1} g_{2}\right)^{\sim} \circ \tilde{g}_{3}=f\left(g_{1}, g_{2}\right) \circ f\left(g_{1} g_{2}, g_{3}\right) \circ\left(g_{1} g_{2} g_{3}\right)^{\sim} \text {. }
$$

But also

$$
\begin{aligned}
\tilde{g}_{1} \circ \tilde{g}_{2} \circ \tilde{g}_{3} & =\tilde{g}_{1} \circ f\left(g_{2}, g_{3}\right) \circ\left(g_{2} g_{3}\right)^{\sim}=\tilde{g}_{1} \circ f\left(g_{2}, g_{3}\right) \circ \tilde{g}_{1}^{-1} \circ \tilde{g}_{1} \circ\left(g_{2} g_{3}\right)^{\sim} \\
& =\left(g_{1} f\left(g_{2}, g_{3}\right)\right) \circ f\left(g_{1}, g_{2} g_{3}\right) \circ\left(g_{1} g_{2} g_{3}\right)^{\sim} .
\end{aligned}
$$

Hence (in additive notation) we have

$$
g_{1} f\left(g_{2}, g_{3}\right)-f\left(g_{1} g_{2}, g_{3}\right)+f\left(g_{1}, g_{2} g_{3}\right)-f\left(g_{1}, g_{2}\right)=\delta f\left(g_{1}, g_{2}, g_{3}\right)=0 .
$$

It follows that $f$ determines an element in $H^{2}(G ; A)$, i.e., an extension $0 \rightarrow A$ 
$\rightarrow \widetilde{G} \rightarrow G \rightarrow 1$ of $G$ by $A$. The group $\widetilde{G}$ can be described as follows. As a set, $\widetilde{G}=A$ $\times G$. The group operation in $\tilde{G}$ is given by

$$
\left(\sigma_{1}, g_{1}\right) *\left(\sigma_{2}, g_{2}\right)=\left(\sigma_{1}+g_{1} \sigma_{2}+f\left(g_{1}, g_{2}\right), g_{1} g_{2}\right), \sigma_{1}, \sigma_{2} \in A ; g_{1}, g_{2} \in G .
$$

Now define the action of $(\sigma, g) \in \tilde{G}$ on $\tilde{X}$ as $(\sigma, g)(\tilde{x})=\sigma \circ \tilde{g}(\tilde{x})$. We have

$$
\begin{aligned}
& \left(\left(\sigma_{1}, g_{1}\right) *\left(\sigma_{2}, g_{2}\right)\right)(\tilde{x}) \\
& \quad=\sigma_{1} \circ g_{1} \sigma_{2} \circ f\left(g_{1}, g_{2}\right) \circ\left(g_{1} g_{2}\right)^{\sim}(\tilde{x})=\sigma_{1} \circ \tilde{g}_{1} \circ \sigma_{2} \circ \tilde{g}_{1}^{-1} \circ \tilde{g}_{1} \circ \tilde{g}_{2}(\tilde{x}) \\
& \quad=\sigma_{1} \circ \tilde{g}_{1} \circ \sigma_{2} \circ \tilde{g}_{2}(\tilde{x})=\left(\sigma_{1}, g_{1}\right)\left(\left(\sigma_{2}, g_{2}\right)(\tilde{x})\right) .
\end{aligned}
$$

That is, this definition makes $\widetilde{G}$ a transformation group on $\tilde{X}$. The other assertions of the theorem can be readily verified.

We are now in the position to discuss free actions of an elementary 2-group on a cohomology projective space $X$.

THEOREM 3.3. If an elementary 2-group $G$ of rank $k$ acts freely on a cohomology projective $n$-space $X$, then the following are true.

(i) If $n$ is even, then $k=0$ (i.e., $G$ is a trivial group).

(ii) If $n$ is odd and $n \neq 4 m-1(m=1,2, \ldots)$, then $k \leqq 1$.

(iii) If $n$ is odd and $n=4 m-1(m=1,2, \ldots)$, then $k \leqq 2$.

Proof. (i) follows from a direct consideration of the Euler characteristic; its proof is omitted here.

According to [8], there exists a cohomology covering space $(\tilde{X}, X, A, \pi), A \cong Z_{2}$, with respect to $\alpha \in H^{1}\left(X ; Z_{2}\right)$, where $\alpha$ is the generator of $H^{1}\left(X ; Z_{2}\right) \cong Z_{2}$ and where $\tilde{X}$ is a $\bmod 2$ cohomology $n$-sphere. Suppose $n \neq 4 m-1$ and $k=2$. By (3.2), there exists an extension $0 \rightarrow A \rightarrow \widetilde{G} \rightarrow Z_{2}^{2} \rightarrow 0$ of $Z_{2}^{2}$ by $A$ and a free action of $\tilde{G}$ on $\tilde{X}$. Since $\tilde{G}$ is of order $2^{3}, \tilde{G}$ is either cyclic or a generalized quaternion group by (3.1). If $\widetilde{G}$ is cyclic, then $\widetilde{G} / A \cong Z_{4}$ which is a contradiction. If $\tilde{G}$ is a generalized quaternion group, then the group $H^{i}\left(\widetilde{G} ; Z_{2}\right),[3]$, is given by

$$
H^{i}\left(\tilde{G} ; Z_{2}\right) \cong\left\{\begin{array}{llll}
Z_{2} & & \text { if } i=4 m, \\
Z_{2} \oplus Z_{2} & \text { if } i=4 m+1, \\
Z_{2} \oplus Z_{2} & \text { if } i=4 m+2, \\
Z_{2} & & \text { if } i=4 m+3
\end{array}\right.
$$

On the other hand, since $\tilde{G}$ acts freely on a mod 2 cohomology $n$-sphere $\tilde{X}$, we must have $H^{i}\left(\widetilde{G} ; Z_{2}\right) \cong H^{i+n+1}\left(\widetilde{G} ; Z_{2}\right)$ which contradicts (1) if $n \neq 4 m-1$. This proves (ii). Finally, suppose $n=4 m-1$ and $k=3$. Again we have an extension $0 \rightarrow A \rightarrow \widetilde{G} \rightarrow Z_{2}^{3} \rightarrow 0$ of $Z_{2}^{3}$ by $A$ and a free action of $\widetilde{G}$ on $\tilde{X}$. $\widetilde{G}$ is again either cyclic or a generalized quaternion group. In either case, $\widetilde{G}$ has at most two generators and therefore so does the quotient group $\widetilde{G} / A \cong Z_{2}^{3}$. But this is clearly a contradiction. 
Notice that the restrictions of $k$ in the above theorem are "best possible." We demonstrate case (iii). Let $Q$ be the 4-dimensional vector space of quaternions and consider $S^{4 m-1}$ as the unit sphere in $Q^{m}$. It is easily seen that the two maps $s$ and $t$ defined on $S^{4 m-1}$ by $s\left(q_{1}, \ldots, q_{m}\right)=\left(i q_{1}, \ldots, i q_{m}\right)$ and $t\left(q_{1}, \ldots, q_{m}\right)=\left(j q_{1}, \ldots, j q_{m}\right)$ generate a free action of the quaternion group (i.e., a generalized quaternion group with $r=3$ ) on $S^{4 m-1}$ and, hence, a free action of $Z_{2} \oplus Z_{2}$ on $P(4 m-1)$ after identifying antipodal points on $S^{4 m-1}$.

The ring structure of the space is definitely required in Theorem 3.3. To see this, consider the $\left(2^{n}-1\right)$-manifold $Y=S^{1} \times S^{2} \times S^{4} \times \ldots \times S^{2^{n-1}}$. Define a free action of an elementary 2-group of rank $n$ on $Y$ by having the nonzero $i$ th component of a group element operate as the antipodal map on the sphere $S^{2^{i-1}}$. It is easily verified that $Y$ has the mod 2 cohomology group structure of $P\left(2^{n}-1\right)$. Using formula (4) of (2.3), it may be established that no more than $n$ copies of $Z_{2}$ may operate freely on a space which has the mod 2 cohomology group structure of $P(n)$. No attempt will be made in this paper to establish a "best possible", result for this case.

The following "effectiveness" lemma will prove quite useful in the remainder of this paper.

LEMMA 3.4. Let $G$ be an elementary p-group operating effectively, but not freely, on a first countable connected cohomology $n$-manifold $X \bmod p$. Consider the set of components $\mathscr{C}=\{C, C$ is a component of $F(K) \mid K$ running through the cyclic subgroups of $G\}$. Suppose $C_{M}$ is a component in $\mathscr{C}$ which is not properly contained by any member of $\mathscr{C}$ and let $K_{M}$ be a cyclic subgroup such that $F\left(K_{M}\right) \supset C_{M}$. Finally suppose that $T$ is a subgroup of $G / K_{M}$ which leaves $C_{M}$ invariant. Then $T$ is effective on $C_{M}$.

Proof. Suppose $T$ is not effective on $C_{M}$. Then there exists a cyclic subgroup $K^{\prime}$ of $G, K^{\prime} \neq K_{M}$ such that $K^{\prime}$ leaves $C_{M}$ pointwise fixed. Hence $F\left(K^{\prime}\right) \supset C_{M}$ and due to the maximality of $C_{M}, C_{M}$ is a component of $F\left(K^{\prime}\right)$. Consider $K^{\prime} \oplus K_{M}$ $\cong Z_{p} \oplus Z_{p}$ operating on $X$. Now $C_{M}$ is a component of $F\left(K^{\prime} \oplus K_{M}\right)$ and is in fact a component of the fixed point set of every cyclic subgroup of $K^{\prime} \oplus K_{M}$. We may now obtain a contradiction by applying (2.1). Let $x \in C_{M}$ and $r=\operatorname{dim}_{p} C_{M}$. But $n-r>0$ and $n(H)=r$ for every subgroup $H$ of index $p$ in $K^{\prime} \oplus K_{M}$.

We shall refer to $C_{M}$ as a maximal component. In applications of Lemma 3.4 it will be understood that we choose $C_{M}$ from among the elements of $\mathscr{C}$ of maximal $\bmod p$ dimension.

THEOREM 3.5. Let $G$ be an elementary 2-group of rank $k$ operating effectively on a first countable generalized cohomology projective $n$-space $X$. Then

$$
k \leqq \begin{cases}n+1 & \text { for } n=1,3, \\ n & \text { otherwise. }\end{cases}
$$


Proof. Due to (3.3), we may assume the action of $G$ is not free. We proceed by induction on $n$. If $n=1$, then $X$ is a circle and $k \leqq 2,[7]$. Consider then the general case where $\operatorname{dim}_{2} X=n, n>1$. Let $C_{M}$ be a maximal component and let $C_{M} \subset F\left(K_{M}\right)$ for $K_{M}$ a cyclic subgroup of $G$. Since $G$ is not free on $X, C_{M}$ is not empty. By Su's result, we have

$$
F\left(K_{M}\right)=C_{M} \cup C^{\prime}
$$

where $\operatorname{dim}_{2} C_{M}+\operatorname{dim}_{2} C^{\prime}=n-1$. We consider two cases.

Case (1). $\operatorname{dim}_{2} C_{M} \neq \operatorname{dim}_{2} C^{\prime}$. This first case will always hold when $n$ is even. Suppose $\operatorname{dim}_{2} C_{M}=n-1$. Then $\operatorname{dim}_{2} C^{\prime}=0$ and $C^{\prime}$ is a point $x_{0}$. Now $G / K_{M}$ leaves $F\left(K_{M}\right)$ invariant and clearly must leave $x_{0}$ stationary. Hence $x_{0}$ is a fixed point of $G$ on $X$, and by (2.2), $k \leqq n$. We may suppose then that $\operatorname{dim}_{2} C_{M} \leqq n-2$. Since $C_{M}$ and $C^{\prime}$ are of different dimensions, $G / K_{M}$ must leave $C_{M}$ invariant. By (3.4), $G / K_{M}$ is effective on $C_{M}$. Therefore, recalling that $C_{M}$ is a generalized cohomology projective space, it follows by the induction hypothesis that

$$
k-1=\operatorname{rank} G / K_{M} \leqq \operatorname{dim}_{2} C_{M}+1 \leqq(n-2)+1 .
$$

Hence $k \leqq n$.

Case (2). $\operatorname{dim}_{2} C_{M}=\operatorname{dim}_{2} C^{\prime}$. Again $G / K_{M}$ which is of rank $k-1$ leaves $F\left(K_{M}\right)$ invariant. By (2.4), there exists a subgroup $T$ of $G / K_{M}$ of rank at least $k-2$ which leaves $C_{M}$ invariant. By (3.4), $T$ is effective on $C_{M}$. Hence again by induction,

$$
k-2 \leqq \operatorname{rank} T \leqq \operatorname{dim}_{2} C_{M}+1=\frac{n-1}{2}+1
$$

Hence $k \leqq(n+5) / 2$. Noting that $(n+5) / 2<n+1$ for $n>3$ and that $n=2$ has already been covered in Case (1), Theorem 3.5 now follows.

The interest of Theorem 3.5 will be established by now showing that it provides "best possible" bounds on $k$. We shall consider $P(n)$ to consist of all pairs of $(n+1)$-tuples $\left\{\left(x_{1}, x_{2}, \ldots, x_{n+1}\right),\left(-x_{1},-x_{2}, \ldots,-x_{n+1}\right)\right\}$ where the $x_{i}$ are real numbers satisfying the relation $\sum_{i=1}^{n+1} x_{i}^{2}=1$. We define an action of an elementary 2-group on $P(n)$ of rank $n$ by exhibiting the action of $n$ generators of the group. Let

$$
\begin{aligned}
& T_{i}\left\{\left(x_{1}, x_{2}, \ldots, x_{n+1}\right),\left(-x_{1},-x_{2}, \ldots,-x_{n+1}\right)\right\} \\
& \quad=\left\{\left(x_{1}, x_{2}, \ldots,-x_{i}, \ldots, x_{n+1}\right),\left(-x_{1},-x_{2}, \ldots, x_{i}, \ldots,-x_{n+1}\right)\right\}, i=1,2, \ldots, n .
\end{aligned}
$$

It is easily verified that the $T_{i}$ generate an elementary 2-group which operates effectively on $P(n)$.

It remains to consider the special cases of $P(1)$ and $P(3)$. Since $P(1)$ is just a circle it is easy to construct an effective action of two copies of $Z_{2}$ on it. For $P(3)$ consider the following four involutions, 


$$
\begin{aligned}
& T_{1}\left\{\left(x_{1}, x_{2}, x_{3}, x_{4}\right), \ldots\right\}=\left\{\left(x_{2}, x_{1}, x_{4}, x_{3}\right), \ldots\right\}, \\
& T_{2}\left\{\left(x_{1}, x_{2}, x_{3}, x_{4}\right), \ldots\right\}=\left\{\left(x_{3}, x_{4}, x_{1}, x_{2}\right), \ldots\right\}, \\
& T_{3}\left\{\left(x_{1}, x_{2}, x_{3}, x_{4}\right), \ldots\right\}=\left\{\left(-x_{1},-x_{2}, x_{3}, x_{4}\right), \ldots\right\} \\
& T_{4}\left\{\left(x_{1}, x_{2}, x_{3}, x_{4}\right), \ldots\right\}=\left\{\left(-x_{1}, x_{2},-x_{3}, x_{4}\right), \ldots\right\} .
\end{aligned}
$$

By verifying that the $T_{i}$ commute and that no nontrivial composition of them operates trivially on $P(3)$, one obtains an effective action of four copies of $Z_{2}$ on $P(3)$. It is interesting to observe that the fixed point set of each cyclic subgroup of this group on $P(3)$ consists of a pair of circles. Therefore the action falls under Case (2) of Theorem 3.5 and the inequality $k \leqq(n+5) / 2$ applies.

4. Lens spaces. A cohomology lens $(2 n+1)$-space $\bmod p(p$ an odd prime) will be a compact space which has the mod $p$ cohomology ring structure of the lens $(2 n+1)$-space, $\mathscr{L}(2 n+1, p)$. A generalized cohomology lens $(2 n+1)$-space $\bmod p$ will be a cohomology lens $(2 n+1)$-space $\bmod p$ which is also a cohomology $(2 n+1)$-manifold $\bmod p$. By a result of $\mathrm{Su}[9 ; 10]$, it follows that if $Z_{p}$ operates on a (generalized) cohomology lens $(2 n+1)$-space $\bmod p$ then the fixed point set is either empty or consists of at most $p$ components $C_{1}, \ldots, C_{s}(s \leqq p)$ where each $C_{j}$ is a (generalized) cohomology lens $\left(2 n_{j}+1\right)$-space $\bmod p$ and $\sum_{j=1}^{s} n_{j}=n-s+1$.

The following is an immediate consequence of (3.1) and (3.2).

THEOREM 4.1. Every elementary p-group operating freely on a cohomology lens space mod $p$ is of rank 1.

Consider $\mathscr{L}(2 n+1, p)$ to consist of all $p$ tuples of the form $\left\{\left(z_{1}, z_{2}, \ldots, z_{n+1}\right)\right.$, $\left.\left(\rho z_{1}, \rho z_{2}, \ldots, \rho z_{n+1}\right), \ldots,\left(\rho^{p-1} z_{1}, \rho^{p-1} z_{2}, \ldots, \rho^{p-1} z_{n+1}\right)\right\}$ where the $z_{i}$ are complex numbers satisfying the relation $\sum_{i=1}^{n+1}\left|z_{i}\right|^{2}=1$ and where $\rho$ is a primitive $p$ th root of unity. It is possible to construct an effective action of an elementary $p$-group of rank $n+1$ on $\mathscr{L}(2 n+1, p)$. Let the $n+1$ generators of the action be given by

$$
\begin{array}{r}
T_{1}\left\{\left(z_{1}, z_{2}, \ldots, z_{n+1}\right), \ldots\right\}=\left\{\left(\gamma z_{1}, \gamma z_{2}, \ldots, \gamma z_{n+1}\right), \ldots\right\} \\
T_{i}\left\{\left(z_{1}, \ldots, z_{i}, \ldots, z_{n+1}\right), \ldots\right\}=\left\{\left(z_{1}, \ldots, \rho z_{i}, \ldots, z_{n+1}\right), \ldots\right\}
\end{array}
$$

where $\gamma$ is a primitive $p^{2}$ th root of unity and $i=2,3, \cdots, n+1$. It can be verified that the $T_{i}$ are of period $p$, commute and do not have any nontrivial relation. We show that we can not have more than $n+1$ copies of $Z_{p}$ operating effectively on $\mathscr{L}(2 n+1, p)$.

THEOREM 4.2. Let $G$ be an elementary p-group of rank $k$ operating effectively on a first countable generalized cohomology lens $(2 n+1)$-space $X \bmod p$. Then $k \leqq n+1$.

Proof. As in Theorem 3.5, we assume that the action of $G$ is not free and proceed by induction on $n$. If $n=0, X$ is again a circle and $k \leqq 1$ by Smith [7]. Suppose then that $n \geqq 1$ and let $C_{M}$ be a maximal component with corresponding cyclic subgroup $K_{M}$. We have, 


$$
F\left(K_{M}\right)=C_{1} \cup C_{2} \cup \ldots \cup C_{s}
$$

where $s \leqq p$, each $C_{j}$ is a generalized cohomology lens $\left(2 n_{j}+1\right)$-space $\bmod p$ and $\sum_{j=1}^{s} n_{j}=n-s+1$. We suppose $C_{M}$ is $C_{1}$ and consider two cases.

Case (1). $s<p$. In this case $G / K_{M}$ must leave $C_{1}$ invariant by (2.4). By (3.4), $G / K_{M}$ is effective on $C_{1}$. Therefore by the induction hypothesis,

$$
k-1=\operatorname{rank} G / K_{M} \leqq n_{1+1} .
$$

Since $\operatorname{dim}_{p} C_{1} \leqq \operatorname{dim}_{p} X-2, n_{1} \leqq n-1$. Hence $k \leqq n+1$.

Case (2). $s=p$. In this case applying (2.4) and (3.4), we obtain a subgroup $T$ of $G / K_{M}$ of rank at least $k-2$ which operates effectively on $C_{1}$. Therefore

$$
k-2 \leqq \operatorname{rank} T \leqq n_{1+1} \leqq n-p+2 .
$$

Since $p \geqq 3, k \leqq n+1$.

\section{REFERENCES}

1. A. Borel et al., Seminar on transformation groups, Princeton Univ. Press, Princeton, N.J., 1960.

2. H. Cartan et al., Seminaire Henri Cartan, 3e. année., Multilith, Paris, 1950/1951.

3. H. Cartan and S. Eilenberg, Homological algebra, Princeton Univ. Press, Princeton, N.J., 1956.

4. P. E. Conner, On the action of a finite group on $S^{n} \times S^{n}$, Ann. of Math. (2) 66 (1957), 586-588.

5. A. Heller, A note on spaces with operators, Illinois J. Math. 3 (1959), 98-100.

6. L. N. Mann, Compact abelian transformation groups, Trans. Amer. Math. Soc. 99 (1961), 41-59.

7. P. A. Smith, New results and old problems in finite transformation groups, Bull. Amer. Math. Soc. 66 (1960), 401-415.

8. J. C. Su, On a problem of P. A. Smith, Bull. Amer. Math. Soc. 67 (1961), 422-424.

9. - Transformation groups on cohomology projective spaces, Trans. Amer. Math. Soc. (to appear).

10. - Transformation groups on cohomology lens spaces and cohomology complex projective spaces, Abstract 582-12, Notices Amer. Math. Soc. 8 (1961), 425-426.

11. R. G. Swan, A new method in fixed point theory, Comment. Math. Helv. 34 (1960), 1-16.

12. H. Zassenhaus, The theory of groups, 2nd ed., Chelsea, New York, 1949.

UNIVERSITY OF VIRGINIA,

Charlottesville, Virginia 